\title{
Particle production and reheating of the inflationary universe
}

\author{
Ian G. Moss* and Chris Graham \\ School of Mathematics and Statistics, Newcastle University, Newcastle Upon Tyne, NE1 7RU, UK
}

(Dated: July 11, 2021)

\begin{abstract}
Thermal field theory is applied to particle production rates in inflationary models, leading to new results for catalysed or two-stage decay, where massive fields act as decay channels for the production of light fields. A numerical investigation of the Boltzmann equation in an expanding universe shows that the particle distributions produced during small amplitude inflaton oscillations or even alongside slowly moving inflaton fields can thermalise.
\end{abstract}

\section{INTRODUCTION}

Inflationary models give a picture of the early universe that has shown spectacular agreement with observation $[1,2,[3]$. All inflationary models require a mechanism for reheating the universe to take it from the vacuum dominated inflationary phase to the hot radiation-dominated universe which we know must follow. The details of particular reheating mechanisms depend on the interactions between the inflaton and other fields, but the underlying process is particle production which fills the universe with radiation.

The original work on reheating in the 1980's introduced particle production in an ad hoc fashion, assuming the rate of particle production by the inflaton $\phi$ in the limit of small $\dot{\phi}$ was proportional to $\dot{\phi}^{n}[4,[5,6]$. Most authors settled for $n=2$, which has the advantage of being equivalent to a simple friction term in the inflaton equation of motion. At around the same time, a less ad hoc approach was based on particle production caused by an inflaton field oscillating about the minimum of the inflaton potential after the end of inflation [7, 8]. This latter approach appeared to be the more consistent, and it is still widely used today.

Following this early work, there where several attempts to apply new ideas in thermal field theory to the reheating process. These usually focused on finding effective field equations for the inflaton. The small- $\dot{\phi}$ equation of motion was derived first, using linear response theory [9, 10]. This has been used in the theory of warm inflation [11, 12, 13]. More general forms of the inflaton field equation, which where not limited to small time derivatives and could be applied to the oscillating inflaton, followed later [14, 15, 16].

Renewed interest in particle production was ignited by the discovery of preheating, a nonperturbative process of inflaton decay though parametric resonance [17, 18, 19, 20, 21]. Like the earlier work, preheating involves particle production from an oscillating inflaton field. Preheating is a result of very large amplitude oscillations in the inflaton field. Large amplitude oscillations are a feature, though not necessarily a desirable one, of most single-field inflationary models.

Preheating may or may not occur depending on the details of the particle model. In this paper we shall focus on models with a mechanism which we call catalysed or two-stage decay [22]. Unlike in the case of pre-heating, we examine what happens when the fields coupled to the inflaton are too massive to be produced directly. Instead, the fields can act as decay channels for the production of light fields. This requires the kind of mass hierarchy which is possible in supersymmetric models. Examples include Grand Unified extensions of the standard model.

In section II we obtain formulae for the particle production rate due to changing particle masses or couplings and show that to a reasonable degree of accuracy the expansion of the universe can be neglected in making these calculations. In section III we consider several models of particle production that could arise during inflation, starting with the oscillating and slowly-evolving models, for which particle production rates are well-known and easily checked, and then we apply our method to the catalysed decay.

In section IV we use the production rates calculated in the previous section to take a closer look at the evolution of a system which may be expanding and departing from thermal equilibrium. Thermalisation has previously been considered in the context of reheating through numerical solution of classical non-linear field theory [23, 24] or using a numerical solution for the non-equilibrium particle propagators [25, 26]. We solve the Boltzmann equation in an expanding universe with a source term representing particle production. This allows us to consider models which would be too difficult to analyse directly using numerical approaches to quantum field theory.

*Electronic address: ian.moss@ncl.ac.uk 


\section{PARTICLE PRODUCTION}

In an ideal situation we would like to track the formation of particles during and after the inflationary era to give a full description of the reheating process. This requires a workable definition of particle number. This definition need not be unique, but at least it should agree with the usual definition of particle number after inflation has ended. We shall use a definition of particle number which introduces a free field which instantaneously has the same field value and momentum as the interacting particle field.

A fundamental problem we face is that the particle production might not have a local description. However, we might hope that simple situations occur where the particles are produced at a rate depending only on local conditions, for example local field values or temperatures. For this reason, we focus on particle production rates.

We shall consider the production of particles due to a background time-dependent inflaton field $\phi$. The particles will be associated with a field $\sigma$.

\section{A. Particle number}

Following Morikawa and Sasaki [10], we define particle creation and anihilation operators for a fiducial free field which coincides with the interacting field $\hat{\sigma}$ and momentum $\hat{\pi}$ at time $t$,

$$
\begin{aligned}
\hat{a}^{\dagger}(p, t) & =\omega_{p} \hat{\sigma}(-p, t)-i \hat{\pi}(p, t) \\
\hat{a}(p, t) & =\omega_{p} \hat{\sigma}(p, t)+i \hat{\pi}(-p, t)
\end{aligned}
$$

where $\omega_{p}=\left(p^{2}+m^{2}\right)^{1 / 2}$ may depend on time, and

$$
\hat{\sigma}(p, t)=\int d^{3} x \hat{\sigma}(x, t) e^{-i p \cdot x} .
$$

The local number density is assumed to be spatially homogeneous. The number density in phase space $n(p)$ can then be defined in terms of an ensemble average using the fiducial free field,

$$
\frac{1}{2 \omega_{p}}\left\langle\hat{a}^{\dagger}\left(p_{1}, t\right) \hat{a}\left(p_{2}, t\right)\right\rangle=(2 \pi)^{3} \delta\left(p_{1}-p_{2}\right) n\left(p_{1}\right) .
$$

We shall express the density function in terms of the Wightman function $G_{21}\left(p, t_{1}, t_{2}\right)$, defined by

$$
\left\langle\hat{\sigma}\left(p_{1}, t_{1}\right) \hat{\sigma}\left(p_{2}, t_{2}\right)\right\rangle=(2 \pi)^{3} \delta\left(p_{1}-p_{2}\right) G_{21}\left(p_{1}, t_{1}, t_{2}\right) .
$$

The expression for the density function becomes

$$
n(p, t)=\left[\frac{1}{2 \omega_{p}}\left(\omega_{p}-i \partial_{t_{1}}\right)\left(\omega_{p}+i \partial_{t_{2}}\right) G_{21}\left(p, t_{1}, t_{2}\right)\right]
$$

where [...] will be used to indicate when a function is to be evaluated at $t_{1}=t_{2}=t$. The frequency $\omega_{p}$ always refers to the value at time $t$, unless we state otherwise.

\section{B. Particle creation}

In order to use Eq. (6), we would have to solve field equations for the Wightman function with suitable initial data, giving a particle density which is typically a non-local function depending on the history of the background field. Instead of working directly with the density function directly, we shall look for a local approximation to the particle production rate.

After some elementary manipulation, the particle production rate in phase space obtained by taking the time derivative of Eq. (6) becomes

$$
\dot{n}=\dot{n}_{\text {mass }}+\dot{n}_{\text {int }}
$$

The first term represents particle production due to the changing particle mass,

$$
\dot{n}_{\text {mass }}=\left[\frac{\dot{\omega}_{p}}{2 \omega_{p}}\left(\left(\omega_{p}-i \partial_{t_{1}}\right)+\left(\omega_{p}+i \partial_{t_{2}}\right)-\frac{1}{\omega_{p}}\left(\omega_{p}-i \partial_{t_{1}}\right)\left(\omega_{p}+i \partial_{t_{2}}\right)\right) G_{21}\left(p, t_{1}, t_{2}\right)\right]
$$


The second term represents particle production at fixed particle mass,

$$
\dot{n}_{\text {int }}=-\left[\frac{i}{2 \omega_{p}}\left(\left(\partial_{t_{1}}^{2}+\omega_{p}^{2}\right)\left(\omega_{p}+i \partial_{t_{2}}\right)-\left(\omega_{p}-i \partial_{t_{1}}\right)\left(\partial_{t_{2}}^{2}+\omega_{p}^{2}\right)\right) G_{21}\left(p, t_{1}, t_{2}\right)\right]
$$

We can put these expressions into a more useful form by introducing the self-energy. Since we are interested in the evolution of operators in a given initial state it proves convenient to use an 'in-in' formalism, and we use the Schwinger-Keldysh version [27, 28]. Propagators carry two extra internal indices $a$ and $b$, where the indices $a$ and $b$ take the values 1 or 2. Following Calzetta and $\mathrm{Hu}[29]$, we raise and lower indices with a metric $c_{a b}=\operatorname{diag}(+1,-1)$.

The Schwinger-Dyson equations for the in-in formalism read [29],

$$
\begin{aligned}
& \left(\partial_{t_{2}}^{2}+\omega_{p}^{2}\left(t_{2}\right)\right) G_{21}\left(p, t_{1}, t_{2}\right)=-\int d t^{\prime} G_{2}^{a}\left(p, t_{1}, t^{\prime}\right) \Sigma_{a 1}\left(p, t^{\prime}, t_{2}\right) \\
& \left(\partial_{t_{1}}^{2}+\omega_{p}^{2}\left(t_{1}\right)\right) G_{21}\left(p, t_{1}, t_{2}\right)=-\int d t^{\prime} \Sigma_{2}^{a}\left(p, t_{1}, t^{\prime}\right) G_{a 1}\left(p, t^{\prime}, t_{2}\right) .
\end{aligned}
$$

For the terms in the particle production rate which have just one time derivative of the propagator, we use the LSZ trick of introducing a time integral

$$
\begin{aligned}
\left.\left(\partial_{t_{2}}-i \omega_{p}\right) G_{21}\left(p, t_{1}, t_{2}\right)\right|_{t_{2}=t} & =\int_{-\infty}^{t} d t_{2} e^{-i \omega_{p}\left(t-t_{2}\right)}\left(\partial_{t_{2}}^{2}+\omega_{p}^{2}\right) G_{21}\left(p, t_{1}, t_{2}\right) \\
\left.\left(\partial_{t_{1}}+i \omega_{p}\right) G_{21}\left(p, t_{1}, t_{2}\right)\right|_{t_{1}=t} & =\int_{-\infty}^{t} d t_{1} e^{-i \omega_{p}\left(t_{1}-t\right)}\left(\partial_{t_{1}}^{2}+\omega_{p}^{2}\right) G_{21}\left(p, t_{1}, t_{2}\right) .
\end{aligned}
$$

We can now express the particle production rate in terms of integrals of the propagator and the self-energy which have a suitable form for applying perturbation theory.

We shall consider the leading order in perturbation theory. First of all, let

$$
\omega_{p}^{2}=p^{2}+m_{\sigma}^{2}+g^{2} \phi^{2}(t)
$$

where $m_{\sigma}$ is a constant and $\phi(t)$ is given. Suppose also that $\Sigma=O\left(g^{4}\right)$. This is the kind of situation would arise, for example, given an inflaton $\phi$ and an interaction Lagrangian density $\mathcal{L}=g^{2} \phi^{2} \sigma^{2} / 4$.

The leading order result, using Eq. (8) and Eqs. (10,13), is that

$$
\dot{n}_{\text {mass }}=\operatorname{Re}\left\{\frac{2 g^{2} \phi \dot{\phi}}{\omega_{p}} \int_{-\infty}^{t} d t_{2} \frac{e^{-i \omega_{p}\left(t-t_{2}\right)}}{2 \omega_{p}}\left(\phi^{2}(t)-\phi^{2}\left(t_{2}\right)\right) G_{21}\left(p, t, t_{2}\right)\right\}
$$

where $G_{21}\left(p, t, t_{2}\right)$ is a free Wightman function for the $\sigma$-field with the shifted mass (which need not be in the vacuum state).

For the next term, we require the derivative of the Schwinger-Dyson equation (10),

$$
\left(\partial_{t_{1}}^{2}+\omega_{p}^{2}\right)\left(\partial_{t_{2}}^{2}+\omega_{p}^{2}\right) G_{21}\left(p, t_{1}, t_{2}\right)=i \Sigma_{21}\left(p, t_{1}, t_{2}\right)+O\left(g^{6}\right) .
$$

Use this together with Eqs. (9), (12) and (13),

$$
\dot{n}_{\text {int }}=\operatorname{Im}\left\{2 \int_{-\infty}^{t} d t_{2} \frac{e^{-i \omega_{p}\left(t-t_{2}\right)}}{2 \omega_{p}} \Sigma_{21}\left(p, t, t_{2}\right)\right\} .
$$

where $\Sigma_{21}$ is the self-energy of the $\sigma$-field at leading order. We can see from this expression that $\dot{n}_{\text {int }}$ is the part of the production rate which is associated with the imaginary part of the self-energy.

\section{Curved space}

The formulae for the particle production rates found in the previous section neglected the expansion of the universe. In this section we shall seek to show that expansion can be neglected to a reasonable degree of accuracy when particle momenta are larger than the expansion rate. 
Consider a spatially flat universe with scale factor $a$ and constant expansion rate $H$. Solutions to the wave equation in de Sitter space can be decomposed into particle modes [30] with comoving wave number $\mathbf{k}$ which satsify

$$
\left(\partial_{t}^{2}+3 H \partial_{t}+\omega_{p}^{2}\right) f(k, t)=0
$$

where

$$
\omega_{p}^{2}=k^{2} / a^{2}+m^{2}+12 \xi H^{2} .
$$

A suitable normalisation is to use $f \dot{f}^{*}-f^{*} \dot{f}=i / a^{3}$. The modes can be expressed in terms of Hankel functions,

$$
f(k, t)=\frac{\sqrt{\pi}}{2} H k^{-3 / 2} z^{3 / 2} H_{\nu}^{(1)}(z),
$$

where $z=k /(H a)$ and $\nu^{2}=9 / 4-m^{2} / H^{2}-12 \xi$.

Consider a non-interacting field with Wightman function

$$
G_{21}\left(k, t_{1}, t_{2}\right)=(N(k)+1) f\left(k, t_{1}\right) f^{*}\left(k, t_{2}\right)+N(k) f\left(k, t_{2}\right) f^{*}\left(k, t_{1}\right) .
$$

The phase space number density is defined as before,

$$
n(p, t)=\left[\frac{a^{3}}{2 \omega_{p}}\left(\omega_{p}-i \partial_{t_{1}}\right)\left(\omega_{p}+i \partial_{t_{2}}\right) G_{21}\left(k, t_{1}, t_{2}\right)\right] .
$$

This is a function of the momentum $\mathbf{p}=\mathbf{k} / a$ of a locally defined flat-spacetime theory. The factor $a^{3}$ is present because the Wightman function has undergone a shift in normalisation due to the use of comoving modes. We substitute the Wightman function (21) and use the modes (20). The result is quite complicated in general, but the main features can be seen in the conformal case $m=0$ and $\xi=1 / 6$. In this case,

$$
n=n_{d e S}+n_{\text {rad }} .
$$

where

$$
\begin{aligned}
n_{d e S} \omega_{p} & =\frac{1}{2} \frac{k}{a}\left(1+\frac{3}{2} \frac{H a}{k}\right)-\frac{1}{2} \omega_{p} \\
n_{\text {rad }} \omega_{p} & =N \frac{k}{a}\left(1+\frac{3}{2} \frac{H a}{k}\right)
\end{aligned}
$$

The non-vanishing contribution to the density function in the de Sitter vacuum is a reflection of the fact that the particle number was defined using the physical momentum. The particle number defined this way is analogous to the response of a particle detector. We can see from Eqs. (24) and (25) that $n \approx N$ for $p>>H$, so that in this limit we recover the flat space results.

Similar considerations apply also to the particle production rates. However, it is important to bear in mind when calculating particle production rates that $\dot{n}$ is evaluated at constant $p$ and not constant $k$,

$$
\left(\frac{\partial n}{\partial t}\right)_{p}=H p\left(\frac{\partial n}{\partial p}\right)_{t}+\left(\frac{\partial n}{\partial t}\right)_{k}
$$

The first term on the right of this equation represents the reduction in particle density caused by the expansion of the universe. The second term, representing the particle production, goes over to the flat space-time results when $p \gg H$. The redshift term is analysed further in Sect. IV]

It is interesting to integrate Eqs. (24) and (25) to get a formula for the energy density.

$$
\rho_{r}=\rho_{d e S}+\int \frac{d^{3} p}{(2 \pi)^{3}} \frac{k}{a} N(k)\left(1+\frac{3}{2} \frac{H^{2} a^{2}}{k^{2}}\right) .
$$

The vacuum energy density of de Sitter $\rho_{d e S}$ space comes from integrating the left hand side of Eq. (24), after we apply suitable regularisation methods. The full expression combines both curved space and thermal effects, with thermal effects dominating the integral for $p>>H$. 


\section{EXAMPLES}

We shall take a closer look at four different models and calculate some particle production rates using the formalism described in the previous section. These models are typical of what one might expect in the context of inflation. Some of these results have been obtained before using other methods, and these are included to check the consistency of the new approach.

\section{A. Oscillating fields}

The first example we consider is the particle production from small amplitude oscillations of an inflaton or other background field. In many inflationary models, this type of particle production would be eclipsed by preheating from large amplitude oscillations. However, this is not always the case, so that even this simple example may be of interest.

The background field we take has

$$
\phi=\phi_{0}\left(1+\epsilon \cos m_{\phi} t\right)
$$

where $\phi_{0}$ is the stable vacuum value of the field and $\epsilon$ is small variable which varies slowly on the oscillation timescale. The field $\phi$ is coupled to a field $\sigma$ with effective frequency $\omega_{p}$,

$$
\omega_{p}^{2}=p^{2}+m_{\sigma}^{2}+g^{2} \phi^{2}-g^{2} \phi_{0}^{2}
$$

In this case we have introduced a shift so that the mass is $m_{\sigma}$ when $\phi=\phi_{0}$.

This particle production problem was solved long ago [31]. The total 2 -particle production rate is given by the standard formula for particle decay,

$$
\dot{N}=\frac{|\mathcal{M}|^{2}}{8 \pi} \frac{p}{m_{\phi}}
$$

where the momentum $p$ is determined by momentum conservation and the reduced matrix element $\mathcal{M}$ is defined by

$$
\left\langle\mathbf{p}_{1}, \mathbf{p}_{2} \mid 0\right\rangle=\mathcal{M}(2 \pi)^{4} \delta\left(\mathbf{p}_{1}+\mathbf{p}_{2}\right) \delta\left(\omega_{p_{1}}+\omega_{p_{2}}-m_{\phi}\right)
$$

To leading order, the interaction with the background through Eq. (29) gives

$$
\dot{N}=\frac{1}{8 \pi} g^{4} \phi_{0}^{4} \epsilon^{2}\left(1-\frac{4 m_{\sigma}^{2}}{m_{\phi}^{2}}\right)^{1 / 2} \quad m_{\phi}>2 m_{\sigma} .
$$

The dependence on the scalar field energy density $\rho_{\phi}=\phi_{0}^{2} \epsilon^{2} m_{\phi}^{2}$ is usually factored to define the reheating coefficient $\Gamma$ by,

$$
\Gamma=\frac{\dot{\rho}_{r}}{\rho_{\phi}} \approx \frac{g^{4} \phi_{0}^{2}}{8 \pi m_{\phi}}
$$

Since $g$ is typically very small, this type of perturbative reheating is quite inefficient and would take several Hubble times to complete.

We can consider the same problem, but using the general result for the time derivative of the density function (8). The free Wightman function for the vacuum state is given by

$$
G_{21}(t)=\frac{1}{2 \omega_{p}} e^{-i \omega_{p} t}
$$

After integration over time, we have

$$
\dot{n}=\frac{\pi}{4} g^{4} \phi_{0}^{4} \epsilon^{2} \frac{m_{\phi}}{\omega_{p}^{3}} \sin ^{2}\left(m_{\phi} t\right) \delta\left(\omega_{p}-m_{\phi} / 2\right)+\frac{1}{4} g^{4} \phi_{0}^{4} \epsilon^{2} \frac{m_{\phi}^{3}}{\omega_{p}^{4}} \frac{\sin \left(2 m_{\phi} t\right)}{\left(4 \omega_{p}^{2}-m_{\phi}^{2}\right)} .
$$

The total particle creation rate integrated over momentum is

$$
\dot{N}=\frac{1}{4 \pi} g^{4} \phi_{0}^{4} \epsilon^{2}\left(1-\frac{4 m_{\sigma}^{2}}{m_{\phi}^{2}}\right)^{1 / 2} \sin ^{2}\left(m_{\phi} t\right)+\frac{1}{8 \pi} g^{4} \phi_{0}^{4} \epsilon^{2} \frac{m_{\phi}^{2}-8 m_{\sigma}^{2}}{m_{\phi} m_{\sigma}} \sin \left(2 m_{\phi} t\right) .
$$

This result appears complicated, but this is due to the presence of transient terms. Such terms are to be expected when we try to calculate the particle production instantaneously. Over several oscillatory cycles, however, the production rate averages out and we recover the scattering theory result (32). The particle production in this case is effectively localised as long as we consider times longer than the oscillatory cycles. 


\section{B. Derivative expansions}

Another situation where we can have localised particle production rates is in the 'adiabatic' limit, when the inflaton has a small time derivative. This is a specialised form of particle production which does not usually occur at leading order in perturbation theory. An important exception occurs when the system starts out and remains close to thermal equilibrium. This type of particle production was first discovered by Hosoya and Sakagama [9] and by Morikawa and Sasaki [10].

We start again from the general result for the particle creation rate (8), using the adiabatic approximation

$$
\delta \phi^{2}\left(t_{2}\right)=\phi^{2}(t)-\phi^{2}\left(t_{2}\right) \approx 2 \phi(t) \dot{\phi}(t)\left(t_{2}-t\right) .
$$

We introduce transforms,

$$
\begin{aligned}
G_{21}\left(t-t_{2}\right) & =\int_{-\infty}^{\infty} \frac{d \omega}{2 \pi} e^{-i \omega\left(t-t_{2}\right)} G_{21}(\omega) \\
\delta \phi^{2}\left(t_{2}\right) & =\int_{-\infty}^{\infty} \frac{d \omega}{2 \pi} e^{i \omega\left(t-t_{2}\right)} \delta \phi^{2}(\omega)
\end{aligned}
$$

After integration, we arrive at

$$
\dot{n}=g^{4} \phi^{2} \dot{\phi}^{2} \frac{G_{21}^{\prime}\left(-\omega_{p}\right)}{\omega_{p}^{2}} .
$$

It only remains to give a formula for the thermal Wightman function. This can be expressed in terms of a spectral function $\rho$ and the thermal distribution function $n$ (for example, see [32]),

$$
G_{21}=-i(1+n) \rho .
$$

The spectral function typically has a Breit-Wigner form [33],

$$
\rho=\left(\omega^{2}-\omega_{p}^{2}-2 i \omega \tau^{-1}\right)^{-1}-\left(\omega^{2}-\omega_{p}^{2}+2 i \omega \tau^{-1}\right)^{-1}
$$

where $\tau$ is known as the relaxation time. Inserting the Wightman function into Eq. (40) gives

$$
\dot{n}=-g^{4} \phi^{2} \dot{\phi}^{2} \frac{\tau n^{\prime}\left(\omega_{p}\right)}{\omega_{p}^{3}} .
$$

This formula can also be derived using the methods of Ref. [10], and it is closely related to the work of Ref. [9].

Note that the particle production is exponentially small for temperatures less than the $\sigma$-particle mass. In fact, Eq. (40) vanishes at zero temperature due to a general property of the Wightman function. The best way to view this type of particle production is as a type of transport phenomenon, similar to thermal or electrical conductivity. Particles are produced as the system responds to the disturbance of thermal equilibrium caused by the changing mass. Increasing the relaxation time $\tau$ gives more time for the mass to change, driving the system further from equilibrium and increasing the particle production.

The particle production takes energy from the inflaton field, and we can ensure energy balance by introducing a friction term $\Gamma \phi$ into the inflaton equation. The total radiation energy is

$$
\rho_{r}=\int \frac{d^{3} p}{(2 \pi)^{3}} n \omega_{p}
$$

The time variation of $\rho_{r}$ contains a term from the time variation of the $\omega_{p}$, which relates to the change with time of the inflaton effective potential, and a $\Gamma \dot{\phi}^{2}$ term, where

$$
\Gamma=\frac{\dot{\rho}_{r}}{\dot{\phi}^{2}}=-g^{4} \phi^{2} \int \frac{d^{3} p}{(2 \pi)^{3}} \frac{\tau n^{\prime}\left(\omega_{p}\right)}{\omega_{p}^{2}} .
$$

The friction coefficient obtained from the particle production formula agrees with the friction coefficient obtained in the inflaton equation of motion using linear response theory [9]. 


\section{Catalysed or two-stage decay}

In the third model of particle creation an oscillating inflaton decays into light thermal scalar particles through an intermediate virtual boson. This is a natural set-up, because many particle models contain both heavy and light fields, with the light particle masses protected by supersymmetry. Particles which couple to the inflaton will tend to be massive, and may well be too heavy to be produced directly by the inflaton oscillations. Preheating is supressed, and we have to rely on perturbative particle production effects. We shall suppose that the model is part of a supersymmetric theory, which protects the flatness of the inflaton potential and the masses of the light fields, doing away with the need to fine-tune their coupling constants.

The mass of the light particles in this example is independent of time, and we use the second formula (9) for the particle production rate. Having a fixed mass removes some of the ambiguities in the definition of the particle number, and leads to a 'cleaner' result.

The heavy field is denoted by $\chi$ and the light field by $\sigma$. The interaction Lagrangian we shall take is

$$
\mathcal{L}_{I}=-\frac{1}{4} g^{2} \phi^{2} \chi^{2}-\frac{1}{2} h m \sigma^{2} \chi-\frac{1}{4 !} \lambda \sigma^{4}
$$

where $g, h, \lambda$ and $m$ are constants. We can choose $m=g \phi_{0}$ by redefining $h$ if necessary. The self-interaction term of the light fields is included to allow them to come to thermal equilibrium. We shall consider particle production into the vacuum and also in the presence of thermal radiation.

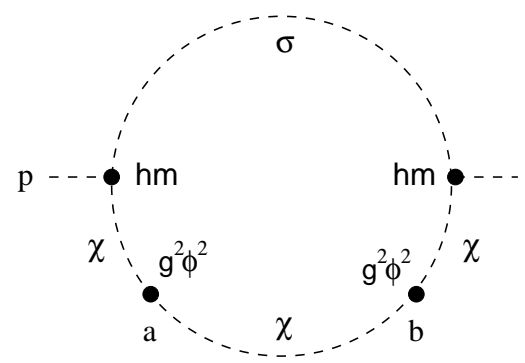

FIG. 1: The Feynman diagram contributing to the imaginary part of the $\sigma$ self energy $\Sigma_{12}$.

The background is as before Eq. (28), but now $m_{\chi}>m_{\phi}$. The first non-trivial contribution to the imaginary part self-energy is given by the Feynman diagram show in figure 1. We define the fourrier transform as in the previous section and then the diagram contributes

$$
\begin{aligned}
& \Sigma_{21}\left(p, t, t_{2}\right)=g^{4} h^{2} m^{2} \int \frac{d^{3} k}{(2 \pi)^{3}} \frac{d \omega_{1}}{2 \pi} \frac{d \omega_{2}}{2 \pi} \frac{d \omega_{3}}{2 \pi} e^{-i \omega_{2}\left(t-t_{2}\right)} G_{\sigma 21}\left(\mathbf{p}-\mathbf{k}, t-t_{2}\right) \\
& G_{\chi 2}{ }^{a}\left(\mathbf{k}, \omega_{1}\right) \phi^{2}\left(\omega_{1}-\omega_{3}\right) G_{\chi a^{b}}{ }^{b}\left(\mathbf{k}, \omega_{3}\right) \phi^{2}\left(\omega_{3}-\omega_{2}\right) G_{\chi b 1}\left(\mathbf{k}, \omega_{1}\right),
\end{aligned}
$$

where a subscript has been used to distinguish between $\chi$ and $\sigma$ propagators.

We concentrate on the low energy spectrum $p<<m_{\chi}$, when we can use a low momentum approximation for the $\chi$ propagators,

$$
\begin{aligned}
G_{\chi 2}^{2}(\mathbf{k}, \omega) & \approx-\frac{i}{m_{\chi}^{2}} \\
G_{\chi 2}^{1}(\mathbf{k}, \omega) & \approx \frac{\alpha}{m_{\chi}^{4}} \theta(\omega) \\
G_{\chi 11}(\mathbf{k}, \omega) & \approx-\frac{i}{m_{\chi}^{2}}
\end{aligned}
$$

where $\theta(\omega)$ is the heaviside function. The middle equation follows from Eqs. (41) and (42), where $\tau^{-1}$ is now the heavy particle decay width and $\alpha=4 \omega_{p} / \tau \propto h^{2} m^{2}$. We use the free Wightman function for the $\sigma$ field with occupation number $n$. 
With these approximations, using Eq. (9) for the particle production rate,

$$
\dot{n}_{i n t}=\frac{g^{2} h^{2}}{2 \pi^{2}} \frac{m^{4} m_{\phi}^{2} \phi_{0}^{2} \epsilon^{2}}{\tau m_{\chi}^{8}} F(p),
$$

where

$$
F(p)=\int \frac{k^{2} d k}{\omega_{k} m_{\phi}^{2}} \theta\left(m_{\phi}-\omega_{p}-\omega_{k}\right)\left(1+n\left(\omega_{k}\right)\right) .
$$

For small $m_{\sigma}$, the integral gives a dilogarithm function,

$$
F(p)=\frac{T^{2}}{m_{\phi}^{2}} \operatorname{dilog}\left(e^{\left(m-\omega_{p}\right) / T}\right) .
$$

The function $F(p)$ has been plotted in figure 2. As might be expected, the vacuum particle production rate peaks when the momentum equals half the inflaton mass. The physical process behind the particle production this time is a decay $\phi \rightarrow 4 \sigma$, using two intermediate virtual $\chi$ bosons. The four particle decay is reflected in the broad width of the peak, compared to the resonance in the 2 -particle decay in Sect. III A.

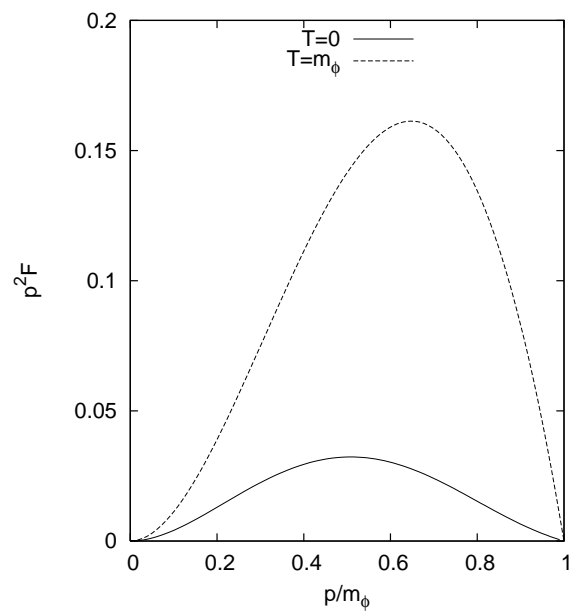

FIG. 2: The momentum dependence of the particle production rate for the two-stage decay with an oscillating inflaton (model C). The function $p^{2} F(p)$ has been plotted for $m_{\sigma}=0$ at $T=0$ and $T=m_{\phi}$.

In the zero temperature limit, the reheating coefficient $\Gamma$ which describes the rate of production of radiation becomes

$$
\Gamma=\frac{\dot{\rho}_{r}}{\rho_{\phi}}=\frac{\alpha g^{2} h^{2}}{480 \pi^{4}} \frac{m^{4} m_{\phi}^{3}}{m_{\chi}^{8}} .
$$

For $\alpha \sim h^{2} m^{2}$, this is smaller than the corresponding result in Sect. IIIA for the $m_{\phi}>m_{\chi}$ regime. However, in supersymmetric models, the couplings do not have to be especially small. Furthermore, if there are many species of light scalar fields, then the the final result scales with the number of fields.

\section{Derivative expansion for catalysed or two-stage decay}

The final example is another 'adiabatic' process, but this time the low temperature behaviour is suppressed by a power law instead of the exponential suppression found in Sect. IIIB. The inflaton decays into light thermal scalar particles through an intermediate virtual boson as in the previous example. This model was introduced in the context of warm inflation [22], but the set-up can occur quite easily in models which contain both heavy and light particles. 
The interaction Lagrangian is the one used in the previous section, Eq. (46). We take an initial state to be one of thermal radiation with temperature $T<<m_{\chi}$. How the system might come to thermal equilibrium will be addressed in the next section.

The first non-trivial contribution to the self-energy is again given by the Feynman diagram show in figure 1 and the expression (47). We can use the condition $T<<m_{\chi}$ to justify a low momentum approximation for the $\chi$ propagators again, now with

$$
\begin{aligned}
G_{\chi 2}^{2}(\mathbf{k}, \omega) & \approx-\frac{i}{m_{\chi}^{2}} \\
G_{\chi 2}^{1}(\mathbf{k}, \omega) & \approx \frac{\alpha}{m_{\chi}^{4}}(1+n) \\
G_{\chi 11}(\mathbf{k}, \omega) & \approx-\frac{i}{m_{\chi}^{2}}
\end{aligned}
$$

where the middle equation follows from Eqs. (41) and (42), with $\alpha=4 \omega / \tau$. In general, $\alpha$ is a function of momentum and energy, which has been calculated explicitly for non-zero temperatures in Ref. 34].

We use an adiabatic approximation for the inflaton fields as in Sect. IIIB.

$$
\delta \phi^{2}(\omega)=2 i \phi \dot{\phi}\left(2 \pi \delta^{\prime}(\omega)\right)
$$

where the primes denote derivatives with respect to $\omega$. With these approximations, the self-energy becomes

$$
\Sigma_{21}\left(p, t, t_{2}\right)=4 g^{4} h^{2} \frac{m^{2} \phi^{2}}{m_{\chi}^{8}} \dot{\phi}^{2} \int \frac{d^{3} k}{(2 \pi)^{3}} \frac{d \omega}{2 \pi} e^{-i \omega\left(t-t_{2}\right)} G_{\sigma 21}\left(\mathbf{p}-\mathbf{k}, t-t_{2}\right)(\alpha(\omega)[1+n(\omega)])^{\prime \prime} .
$$

Now we can apply formula (9) for the particle production rate, using the free thermal propagator for the $\sigma$ field,

$$
\dot{n}_{i n t}=-4 g^{4} h^{2} \frac{m^{2} \phi^{2}}{\omega_{p} m_{\chi}^{8}} \dot{\phi}^{2} \int \frac{d^{3} k}{(2 \pi)^{3}} \frac{\alpha}{\omega_{k}}\left\{n^{\prime \prime}\left(-\omega_{k}-\omega_{p}\right)\left(1+n\left(\omega_{k}\right)\right)\right\} .
$$

So far, we have not had to assume a particular form for the distribution function $n$. However, if $n$ is the thermal distribution function, then the integral can be done approximately in the small $m_{\sigma}$ mass limit,

$$
\dot{n}_{\text {int }}=\frac{g^{2} h^{2}}{2 \pi^{2}} \frac{m^{4} \dot{\phi}^{2}}{\tau m_{\chi}^{8}} F(p)
$$

where $m=g \phi$ and

$$
F(p)=T^{2}\left\{n\left(\omega_{p}\right) \sum_{n=1}^{\infty} \frac{1}{n^{2}}\left(1-e^{-n \omega_{p} / T}\right)\right\}^{\prime \prime} .
$$

The function $F(p)$ has been plotted in figure 3, Note that the pre-factor is identical with the pre-factor in Eq. (51) for the oscillating case if we use the period averaged value of $\dot{\phi}^{2}$. The momentum distribution is quite different, however, and the particle production vanishes as $T \rightarrow 0$.

The reheating coefficient $\Gamma$ can be obtained by integrating over momentum, using $\alpha=4 \omega_{p} / \tau \approx$ const,

$$
\Gamma=\frac{\dot{\rho}_{r}}{\dot{\phi}^{2}}=\frac{\alpha g^{2} h^{2}}{4 \pi^{4}} \frac{m^{4}}{m_{\chi}^{8}} T^{3}
$$

This agrees with the friction coefficient calculated from the effective field equations in ref. [34].

\section{THERMALISATION}

The adiabatic particle production rates where calculated under the assumption that the system was close the thermal equilibrium. In this section we shall examine the validity of this assumption by solving the Boltzmann equation in an expanding universe. This will also give us an opportunity to consider systems which depart from equilibrium. 


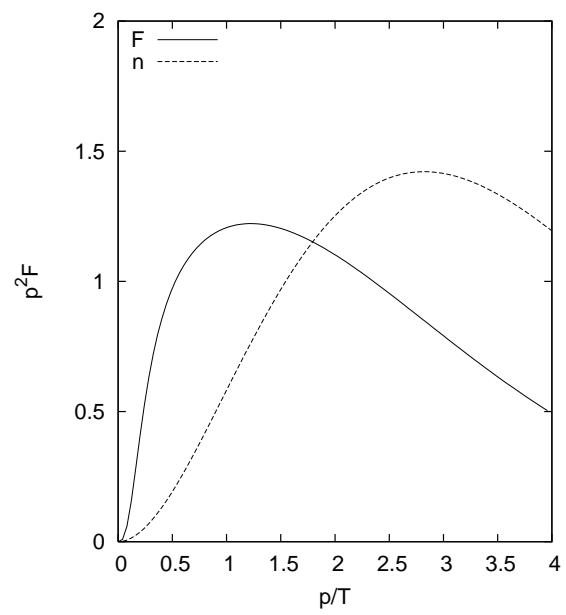

FIG. 3: The momentum dependence of the particle production rate for small $\dot{\phi}$ with the two-stage decay (model D). The function $p^{2} F(p)$ is plotted. The thermal distribution $p^{2} n$ is shown for comparison.

We shall adopt a pseudo-particle approximation where the Wightman function take a thermal form, but with an arbitrary distribution function $n \equiv n(p, t)$. The particle number will evolve according to

$$
\dot{n}=\mathcal{S}_{p}+\mathcal{S}_{r}+\mathcal{S}_{c}
$$

where $\mathcal{S}_{p}$ represents particle production, $\mathcal{S}_{r}$ represents particle dilution due to the expansion of the universe and $\mathcal{S}_{c}$ is the Boltzmann collision term

The particle production rates calculated in Sects. IIIB IIID are still valid with the new distribution functions and can be used for $\mathcal{S}_{p}$. The expansion effect represents a stretching of the physical wavelengths of the modes by the scale factor $a$. This term was evaluated in Eq (26),

$$
\mathcal{S}_{r}=H p \partial_{p} n
$$

The collision term for $2 \rightarrow 2$ particle scattering from the quadratic term in the Lagrangian density Eq. (46) is

$$
\mathcal{S}_{c}=\frac{\lambda^{2}}{2 \omega_{p}} \int \frac{d^{3} p_{2}}{2 \omega_{p_{2}}} \frac{d^{3} p_{3}}{2 \omega_{p_{3}}} \frac{d^{3} p_{4}}{2 \omega_{p_{4}}}(2 \pi)^{-5} \delta\left(P+P_{2}-P_{3}-P_{4}\right) B\left(p, p_{1}, p_{2}, p_{3}\right)
$$

where $P=\left(\omega_{p}, \mathbf{p}\right)$ and

$$
B\left(p, p_{1}, p_{2}, p_{3}\right)=(1+n(p))\left(1+n\left(p_{2}\right)\right) n\left(p_{3}\right) n\left(p_{4}\right)-n(p) n\left(p_{2}\right)\left(1+n\left(p_{3}\right)\right)\left(1+n\left(p_{4}\right)\right) .
$$

This term preserves the total particle number as well as the total energy.

Multiplying the Boltzmann equation by $\omega_{p}$ and integrating gives the total energy equation

$$
\dot{\rho}_{r}+4 H \rho_{r}=S,
$$

where the source term is

$$
S=\int \frac{d^{3} p}{(2 \pi)^{3}} \mathcal{S}_{p} \omega_{p}
$$

In the oscillating inflaton case $S=\Gamma \rho_{\phi}$ and in the slowly evolving inflaton limit $S=\Gamma \dot{\phi}^{2}$, where expression have been given for $\Gamma$ in Sects. IIIB IIID Both types of reheating coefficient can be combined into

$$
\dot{\rho}_{r}+4 H \rho_{r}=\Gamma\left(\rho_{\phi}+p_{\phi}\right),
$$

where $p_{\phi}$ is the averaged pressure term. Various forms of this equation have been used in the past to study reheating [6] and warm inflation [35]. 
The simplest way to analyse the Boltzmann equation (64) is to take a close-to-equilibrium approximation, introducing a thermal distribution function $n_{T}$ and defining the effective temperature by matching the energy density with the actual distribution function,

$$
\int \frac{d^{3} p}{(2 \pi)^{3}}\left(n-n_{T}\right) \omega_{p}=0 .
$$

We can use the thermal particle production rates calculated earlier and introduce a thermal relaxation-time $\tau_{r}$ to simplify the collision term. The Boltzmann equation we have to solve is then

$$
\dot{n}=R F(p)+H p \partial_{p} n-\tau_{r}^{-1}\left(n-n_{T}\right),
$$

where $R$ is the prefactor in the particle production rates Eq. (51) or Eq. (61). We have integrated this equation numerically using a fourth order Runge-Kutta scheme for the time derivatives and second order differences for the momentum derivatives. This numerical procedure is very fast and stable, with most of the results given below taking less than one second on a $1 \mathrm{GHz}$ processor.

\section{A. Oscillating phase}

We consider a period of reheating with an oscillating inflaton and $\rho_{\phi}>>\rho_{r}$. This regime ends, according to Eq. (70), when $H \approx \Gamma$. During this period, the pressure averages to zero over the oscillation period and the universe expands like a pressure free cosmological model, with

$$
\begin{aligned}
& H(t)=H(0)\left(1+\frac{3}{2} H(0) t\right)^{-1} \\
& R(t)=R(0)\left(1+\frac{3}{2} H(0) t\right)^{-2}
\end{aligned}
$$

The second equation follows from $R \propto \rho_{\phi}$.

Some numerical results for the momentum distribution obtained from Eq. (72) are shown in figure 4 , The distribution thermalises, and does so more quickly with smaller relaxation times as might be expected. The momentum distribution of the source term shows up clearly at early times, before the relaxation has taken effect.
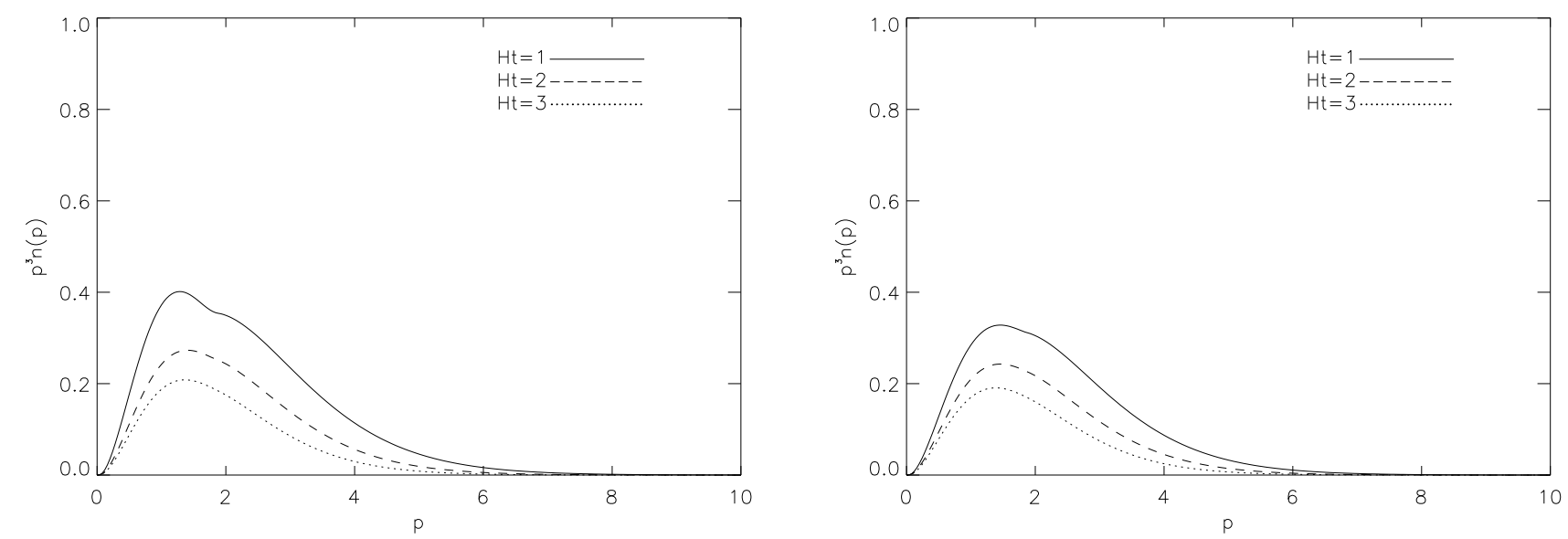

FIG. 4: The stationary momentum distribution for the oscillatory phase in the two-stage decay (model D) using the relaxationtime approximation. The relaxation times are $\tau_{r}=0.1 / H(0)$ (left) and $\tau_{r}=0.05 / H(0)$ (right). As might be expected, shorter relaxation times produce a spectrum which is closer to thermal equilibrium. The constant $R(0)=50 H(0)$ and $m_{\phi}=2 H(0)$. 


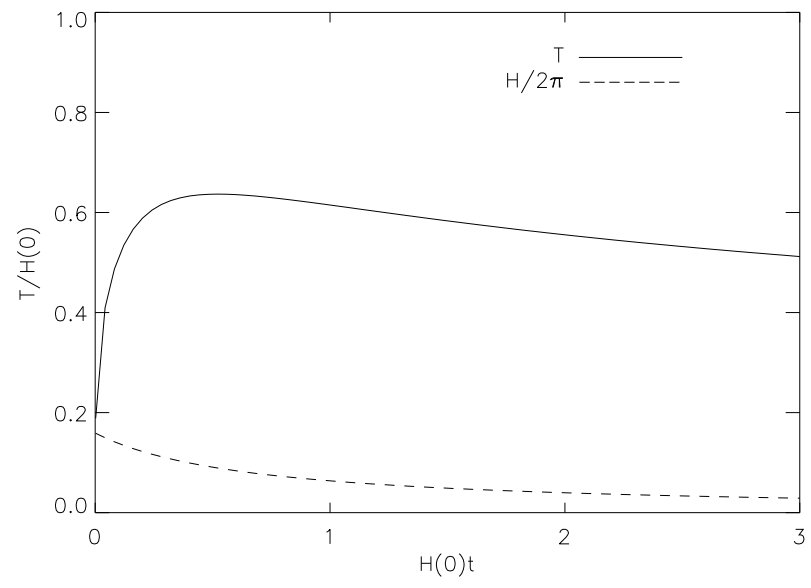

FIG. 5: The time evolution of the effective temperature for the oscillatory phase with the two-stage decay (model D) using the relaxation-time approximation. The de Sitter temperature $H / 2 \pi$ is shown for comparison. The relaxation time $\tau_{r}=0.1 / H(0)$, the constant $R(0)=50 H(0)$ and $m_{\phi}=2 H(0)$.

The initial temperature for the numerical solutions has been set equal to the de Sitter temperature $H(0) / 2 \pi$, to be consistent with the assumptions used in the particle production calculations. The evolution of the temperature is shown in figure 5. After a sharp rise to a maximum, the temperature falls off as $t^{-1 / 4}$. This agrees very well with the analytic solution to the total energy equation (70) [6, 36].

\section{B. Slow-roll phase}

Small values of $\dot{\phi}$ are characteristic of the slow-roll phase of inflation. The particle production rates calculated in sect IIID can be applied to the slow-roll phase, provided we can justify the thermal hypothesis which was used. During the slow-roll phase of inflation, both $H$ and $\Gamma$ vary very little over several Hubble times, and we can treat them as constants in the Boltzmann equation (72).
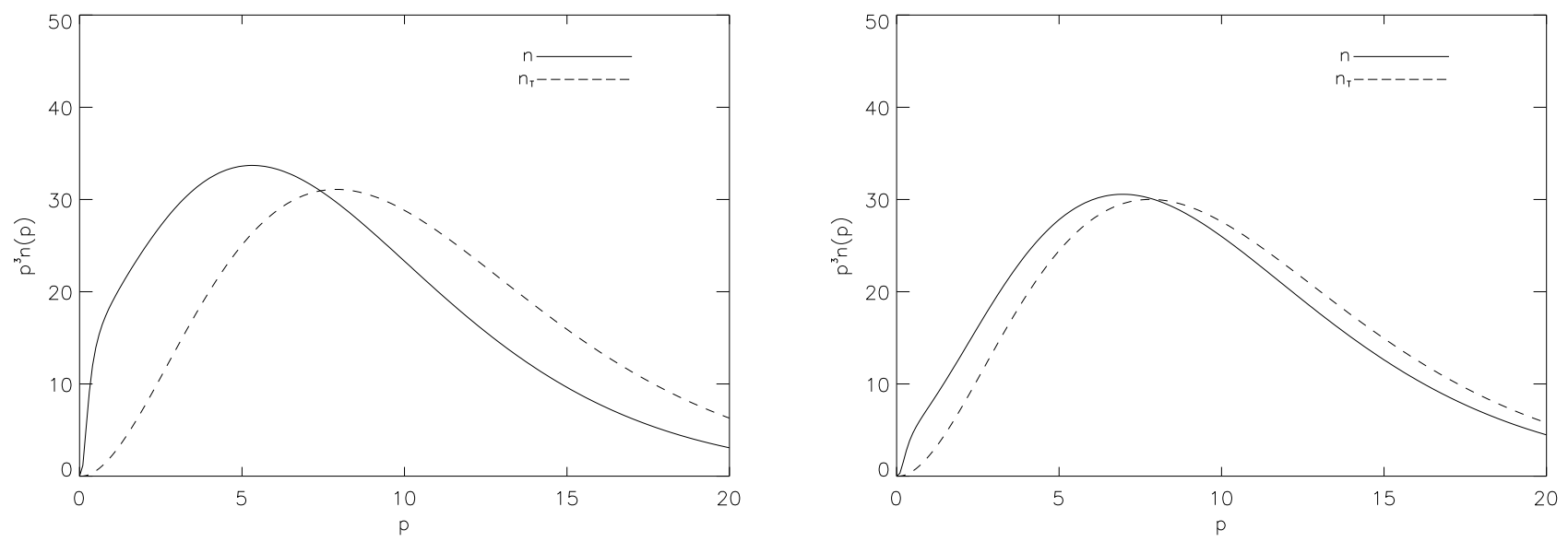

FIG. 6: The stationary momentum distribution for different relaxation times in the two-stage decay (model D) using the relaxation-time approximation. The relaxation times are $\tau_{r}=0.2 / H$ (left) and $\tau_{r}=0.05 / H$ (right). As might be expected, shorter relaxation times produce a spectrum which is closer to thermal equilibrium. The constant $R=15 H$ and $m_{\sigma}=0.25 H$. 


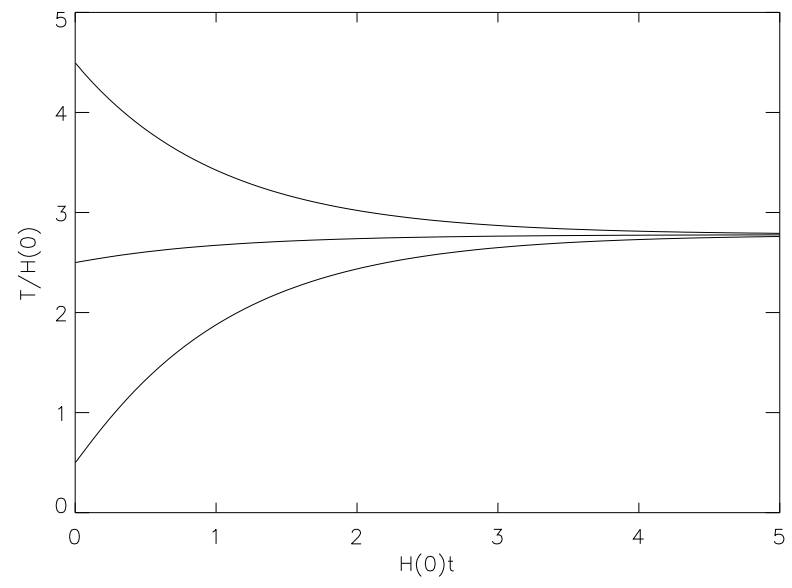

FIG. 7: The time evolution of the effective temperature for different initial conditions with the two-stage decay (model D) using the relaxation-time approximation. In each case, the momentum distribution reaches a stationary state with the effective temperature shown. The relaxation time $\tau_{r}=0.1 / H$, the constant $R=15 H$ and $m_{\sigma}=0.25 H$.

Numerical solutions for two different parameter sets are shown in Fig. 6. These show the existence of an attractor with non-zero temperature and a spectrum close to thermal equilibrium. The final momentum distribution does not show any dependence on the initial distribution, but it is dependent on the relaxation time, a shown in figure6. Small relaxation times, corresponding to relatively large values of the self-coupling $\lambda$, lead to nearly thermal spectra.

The parameters for the numerical solution where chosen to place the temperature in the range $T>H$ required for consistency of the particle production calculations. The time evolution of the temperature shown in figure 7 agrees very well with the analytic solution to Eq. (70) when $\Gamma \propto T^{3}$ (see Eq. (63)), which has the form

$$
T=T_{\infty}\left(1-e^{-H t}\right) .
$$

This shows clearly how the expansion of the inflationary universe need not lead to a supercooled state when particle production is taken into account.

\section{Thermalisation with the full boltzmann collision integral}

In the above work we have introduced the thermal relaxation-time $\tau_{r}$ to approximate the thermalisation effects of the Boltzmann collision term. We can check the validity of this approximation by solving the the Bolzman equation with the full $2 \rightarrow 2$ particle scattering term (66). Following the work of Refs. [31, 37], we can eliminate the delta-functions and reduce the integral from 9 to 2 dimensions, which gives

$$
\mathcal{S}_{c}=\frac{D}{\omega_{p} p} \int \theta\left(\omega_{p_{2}}-m_{\sigma}\right) \min \left(p, p_{2}, p_{3}, p_{4}\right) B\left(p, p_{2}, p_{3}, p_{4}\right) d \omega_{p_{3}} d \omega_{p_{4}},
$$

where $D=\lambda^{2} / 64 \pi^{3}$ and $\omega_{p_{2}} \equiv \omega_{p_{2}}\left(p, \omega_{p_{3}}, \omega_{p_{4}}\right)$ is obtained from energy conservation,

$$
\omega_{p_{2}}=\omega_{p_{3}}+\omega_{p_{4}}-\omega_{p}
$$

We have solved equation (72) numerically with the new expression for $\mathcal{S}_{c}$, focussing on the two stage decay model (model D). Again, we used a fourth order Runge-Kutta scheme for time derivatives and second order differences for the momentum derivatives. The collision term was evaluated using a 2D Simpson's rule integrator. In order to remove instability problems at low momenta we damped the source term with a factor $p^{2} /\left(p^{2}+H^{2}\right)$, which is consistent with our calculation of the source term which cannot be used for $p$ less than $H$. We also avoided using a very fine momentum mesh that would bring in grid points at very low momentum. Solving with the full collision term is computationally far more demanding than using a relaxation-time approximation. For reasonable mesh sizes the total integration times are approximately an hour on $\mathrm{GHz}$ processors, compared to one second for the relaxation-time approximation. 
Numerical results for the full collision term with two stage decay model are shown in figures 8 and 9 , obtained using the same values for constants $R$ and $m_{\sigma}$ as before. The distribution reaches a stable non-zero temperature as expected and is consistent with the findings using the relaxation-time approach.

Comparison of Fig. 6 and Fig. 8 suggests that the relaxation-time $\tau_{r}=0.1 H^{-1}$ corresponds to $D \approx 10$. This example is strongly self-coupled. However, it is possible to argue that value of $D$ needed for thermalisation decreases if we increase the particle production rate. According to dimensional analysis, the relaxation time should be proportional to the inverse temperature. The numerical example has $T=2 H$, hence $D \approx 2 /\left(T \tau_{r}\right)$. We therefore predict a similar distribution function to Fig. 8 for $D<1$ when the particle production is increased to give an effective temperature $T>20 H$.

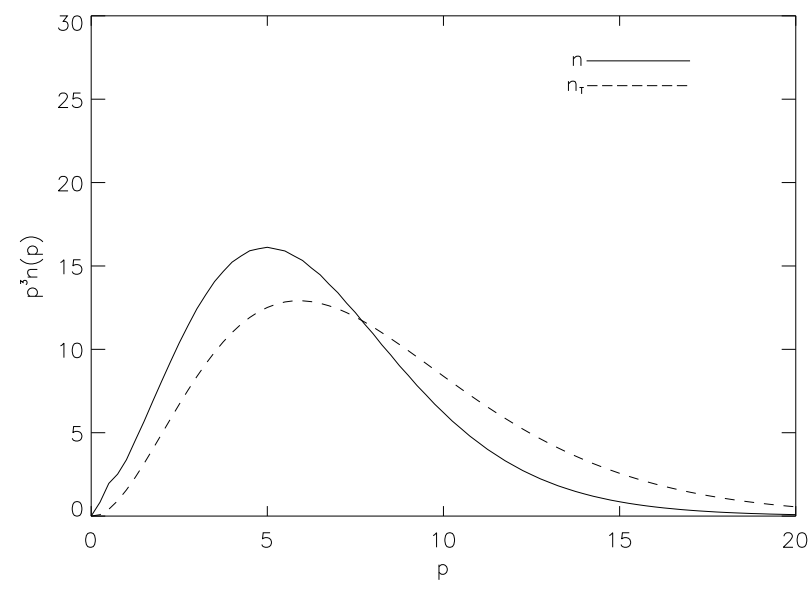

FIG. 8: The stationary momentum distribution in the two-stage decay (model D) using the full collision integral gives a check for consistency of the relaxation time approximation used in Fig. 6. The parameters are $R=15 H$ and $m_{\sigma}=0.25 H$ and $D=10$. The plot is comparable to Fig. [6] with a relaxation time $\tau_{r}=0.1 / H$.

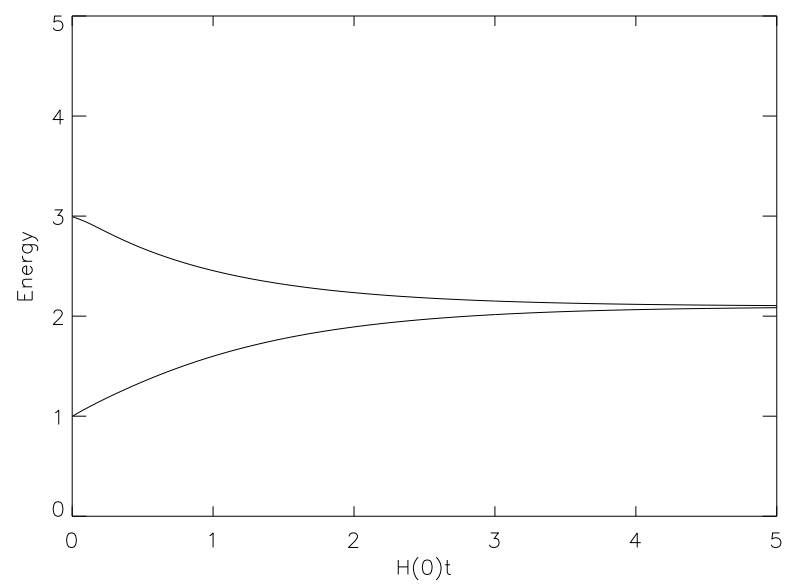

FIG. 9: The time evolution of the effective temperature for different initial conditions with the two-stage decay (model D) using the full collision integral. As with the relaxation time approximation, the momentum distribution reaches a stationary state with the effective temperature shown. The constant $D=10$, with $R=15 H$ and $m_{\sigma}=0.25 H$. 


\section{CONCLUSION}

We have attempted to produce a uniform description of particle production during the early universe which can cope with oscillating and slowly varying inflaton background fields. We have concentrated mainly on a two-stage decay process where the inflaton decays into light radiation fields through an intermediate heavy boson.

Thermalisation of the particles has been described by solving the Boltzmann equation in an expanding universe. We have found that the thermisation and particle production can be combined to produce a prediction for the momentum distribution in the radiation fields. In many cases, where the self-interactions allow, the distribution approaches a thermal distribution.

Our results for particle creation and thermalisation in the case of a slowly-evolving inflaton field are fully consistent with the thermal dissipation processes predicted in warm inflation [33, 34]. Most of these models have have assumed that the radiation remains close to thermal equilibrium, and we have found that this occurs when the the self-coulping of the radiation field is sufficiently large.

The particle production rates, and therefore the thermal dissipation rates, are still significant even when the the distribution function departs substatially from thermal equilibrium. Distortions to the spectra due to the finite relaxation time of the radiation may have observational consequences if the thermal fluctuations are the source of density fluctuations in the cosmic microwave background [11, 38, 39, 40]. Further work would be worthwhile to find the effect this may have on the spectrum and as a source of non-gaussianity.

The reason for considering the two-stage decay lay partly in the fact that there are light fields whose masses are protected by supersymmetry. In a supersymmetric model, the bosonic decays which we have considered would be accompanied by fermionic decays. The extension of the present results to fermions is tedious, but straightforward. Fortunately, fermion decays tend to be suppressed at low temperatures, compared to the bosonic ones [34], and so it should be reasonable to ignore them.

An interesting regime occurs when the temperature is comparable to the expansion rate. Both the thermal equilibrium and flat-spacetime approximations break down in this limit. We have suggested ways to deal with this case using curved space methods in Sect. II C, but further work along these lines would be of interest.

\section{Acknowledgments}

We are grateful to Nick Proukakis and Stuart Coburn for discussions on the Boltzmann equation.

[1] A. H. Guth, Phys. Rev. D 23, 347 (1981).

[2] A. Linde, Phys. Lett. 108B, 389 (1982).

[3] A. Albrecht and P. J. Steinhardt, Phys. Rev. Lett. 48, 1220 (1982).

[4] A. Albrecht, P. J. Steinhardt, M. S. Turner, and F. Wilczek, Phys. Rev. Lett. 48, 1437 (1982).

[5] L. F. Abbott, E. Farhi, and M. B. Wise, Phys. Lett. B117, 29 (1982).

[6] M. S. Turner, Phys. Rev. D 28, 1243 (1983).

[7] A. D. Dolgov and A. D. Linde, Phys. Lett. B116, 329 (1982).

[8] Q. Shafi and A. Vilenkin, Phys. Rev. Lett. 52, 691 (1984).

[9] A. Hosoya and M. A. Sakagami, Phys. Rev. D 29, 2228 (1984).

[10] M. Morikawa and M. Sasaki, Prog. Theor. Phys. 72, 782 (1984).

[11] I. G. Moss, Phys. lett. 154B, 120 (1985).

[12] J. Yokoyama and K. Maeda, Phys. lett. B 207, 31 (1988).

[13] A. Berera, Phys. Rev. lett. 75, 3218 (1995).

[14] D. Boyanovsky, H. J. de Vega, and R. Holman, Phys. Rev. D49, 2769 (1994), hep-ph/9310319.

[15] M. Gleiser and R. O. Ramos, Phys. Rev. D 50, 2441 (1994).

[16] D. Boyanovsky, H. J. de Vega, R. Holman, D. S. Lee, and A. Singh, Phys. Rev. D51, 4419 (1995), hep-ph/9408214.

[17] L. Kofman, A. D. Linde, and A. A. Starobinsky, Phys. Rev. Lett. 73, 3195 (1994), hep-th/9405187.

[18] L. Kofman, A. D. Linde, and A. A. Starobinsky, Phys. Rev. D56, 3258 (1997), hep-ph/9704452.

[19] L. Kofman (1997), hep-ph/9802285.

[20] J. H. Traschen and R. H. Brandenberger, Phys. Rev. D42, 2491 (1990).

[21] Y. Shtanov, J. H. Traschen, and R. H. Brandenberger, Phys. Rev. D51, 5438 (1995), hep-ph/9407247.

[22] A. Berera and R. O. Ramos, Phys. Lett. B 567, 294 (2003).

[23] C. Destri and H. J. de Vega, Phys. Rev. D73, 025014 (2006), hep-ph/0410280.

[24] R. Micha and I. I. Tkachev, Phys. Rev. D70, 043538 (2004), hep-ph/0403101.

[25] G. Aarts and A. Tranberg, Phys. Lett. B650, 65 (2007), hep-ph/0701205. 
[26] G. Aarts and A. Tranberg, Phys. Rev. D77, 123521 (2008), 0712.1120.

[27] J. Schwinger, J Math Phys 2, 407 (1961).

[28] L. V. Keldysh, Zh. Eksp. Teor. Fiz. 47, 1515 (1964).

[29] E. Calzetta and B. L. Hu, Phys. Rev. D 37, 2878 (1988).

[30] N. D. Birrell and P. C. W. Davies, Quantum fields in curved space (Cambridge, UK: Univ. Pr., 1982).

[31] A. D. Dolgov, S. H. Hansen, and D. V. Semikoz, Nucl. Phys. B543, 269 (1999), hep-ph/9805467.

[32] A. Berera, I. G. Moss, and R. O. Ramos (2008), 0808.1855.

[33] A. Berera, M. Gleiser, and R. O. Ramos, Phys. Rev. D 58, 123508 (1998).

[34] I. G. Moss and C. Xiong (2006), hep-ph/0603266.

[35] A. Berera, Phys. Rev. D 55, 3346 (1997).

[36] D. J. H. Chung, E. W. Kolb, and A. Riotto, Phys. Rev. D60, 063504 (1999), hep-ph/9809453.

[37] M. J. Bijlsma, E. Zaremba, and H. T. C. Stoof, Phys. Rev. A62, 063609 (2000), cond-mat/0001323.

[38] A. Berera and L. Z. Fang, Phys. Rev. lett. 74, 1912 (1995).

[39] A. N. Taylor and A. Berera, Phys. Rev. D 62, 083517 (2000).

[40] L. M. H. Hall, I. G. Moss, and A. Berera, Phys. Rev. D69, 083525 (2004), astro-ph/0305015. 